\title{
Efficient GSM based IVRS based Wireless Automation
}

\author{
D. Prasad \\ Professor, Department of ECE, Ramanandatirtha Engineering College, Nalgonda, India
}

\begin{abstract}
After a review of the recent advancements in wireless automation systems, the paper introduces an efficient and economical automation system based on GSM technology. The obtained parameters are processed and recorded in the system memory. If any abnormality or an emergency situation occurs the system sends SMS (short message service) messages to the mobile phones containing information about the abnormality according to some predefined instructions programmed in the microcontroller. This mobile system will help the transformers to operate smoothly and identify problems before any catastrophic failure. The system also permits the user direct devices through his mobile by sending voice commands using IVRS system. It also enables the user to monitor the status of loads with SMS update.
\end{abstract}

Keywords: Automation, mobile, control, GSM (Global System for Mobile Communication), Interactive Voice Response System (IVRS), wireless network, SMS (Short Messaging Service).

\section{Introduction}

Within the ambit of wireless technology, emergence of remote control based devices and appliances have become the order of the day. The scope of such applications ranges from a remote controlled small car toy or a TV to a system that can control a whole building or a factory. Such systems are referred to as Automation Systems. Automation Systems perform by allowing a number of devices to communicate with a central controller which in turn communicates all information to the user or the owner of the system as per the instructions and the structure of the system. The application of such automation systems could be in areas such as heating, lighting, security, energy management, audio and video systems, health monitoring, entertainment and so on.

As part of remote control process of automation systems, there are various communication links that can be used such as RF, Bluetooth, Wi-Fi, Infrared, etc. In this context, this paper proposes a novel method of automation System based on GSM technology. GSM is an open, digital cellular technology used for transmitting mobile voice and data services. GSM supports $9600 \mathrm{kbps}$ band rate for voice calls and data transfers and provides a global range of transmission. With this feature in view, the paper attempts to design a system that can interpret SMS messages and process them effectively, resulting in creating an Automation System that can be controlled wirelessly from anywhere in the world. Such automated systems deals with providing a network in an environment which links computers peripheral equipment, smart chip bearing appliances and sub-systems. With such deployed system, it promises controlling of electricity operated devices cautiously in real time even being physically away from the site. But again these implementations require either radio link communication or wired communication to direct the devices to operate accordingly. Be it wired or radio link communication, each has its own limitations such as complex wire deployment, maintenances, range restrictions, low data rate, high cost, software arrangements etc.

Hence we have implemented the GSM based IVRS incorporated system which enables user to control and monitor the loads/appliances remotely. The GSM based home automation system is developed and implemented which exploits the well-developed infrastructure of GSM cellular system. Moreover, the user do not require dedicated transceiver as the GSM mobile can be used as commanding device. As a result, no extra device has to be purchased from the user side. The GSM network that relies on radio wave communication conveying information in a real-time process to control devices remotely is the most apt alternative among all the wireless technologies. The IVRS system enables user to interact with system which eases the control operation.

\section{Literature Review}

In a study carried out by Delgado et al [1], the authors have examined the possible of remote controlled Home Automation Systems and their welfares. Separately from this, the paper emphasized certain precarious issues that have to be careful while evolving a Home Automation System which range from trust worthiness, robustness, helpfulness, price to security factors. The study familiarized an estimation methodology for user interface grounded on certain aspects.

In alternative study [2], the authors have considered and implemented an internet based home automation system through wireless communication. The study viewed that the main purpose of home automation is to control home devices from a central control point. The study demonstrated that when the control of device is completely governed by machines, the operation of monitoring and reporting becomes essential. The system included a web page implemented on a web server used as an interface to control a number of devices and from which the whole system can be monitored. This module is connected to a master node through an RS232 cable. The master node controls a set of connected devices through RF link.

In yet another paper [3], the authors have introduced a Bluetooth based home automation system. The paper 


\section{International Journal of Science and Research (IJSR) \\ ISSN (Online): 2319-7064 \\ Index Copernicus Value (2013): 6.14 | Impact Factor (2014): 5.611}

described a system that comprises a mobile host controller connected to a number of client modules through Bluetooth. The host controller is a PC with Bluetooth capability and a preinstalled user interface.

A similar study [4] presented a low-cost, expandable and secure Bluetooth based home automation system. The design was based on an Arduino BT board along with a set of appliances connected to it through relays. The host unit in the design was cell phone, instead of PC unit. A GUI was developed using Python script which is compatible with all Symbian OS platforms.

The script allows the user to access and control a set of home appliances. Hence, this limits the use of the system to only cell phones with Symbian operating system.

In another study, [5] the authors have designed and developed a simple and cost-effective automation system that allows individuals to manage home appliances from a computer or a handheld device.

\section{System Methodology}

The system consists of two main sections namely; the transmitter side Commanding Unit (CU) which is basically a GSM enabled mobile phone and the receiver side Appliance Control Unit (ACU). The system is as depicted in Fig.1. The ACU manages devices and loads those need to be monitored and controlled through IVRS voice commands.

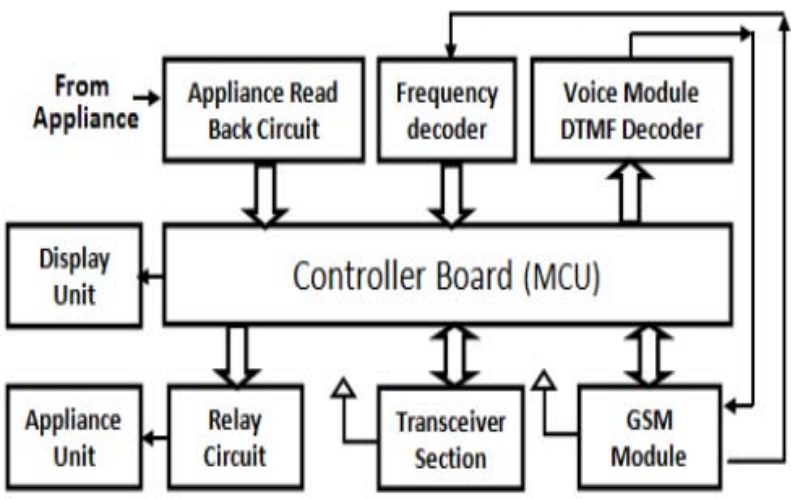

Figure 1: Block Diagram of the ACU System

A. GSM Module: In our scheme GSM sim300 module [5] is used which is top in application such as remote control, SMS alerts, sensor monitoring etc. The GSM module is interfaced with MCU viaRS232 interface. SIM300 designed for global market is a Tri-band GSM/GPRS engine which works on the frequencies $900 \mathrm{MHz}$ EGSM, $1800 \mathrm{MHz}$ DCS and $1900 \mathrm{MHz}$ PCS. SIM300 features GPRS multi slot class10/ class8 and supports the GPRS coding schemes CSi,CS-ii,CS-iii and CSiv. The fundamental two purposes of this unit are:

- Allows the user to interact with the ACU by GSM network radio link.

- Delivers SMS update regarding Appliance switch ON/OFF status and Electricity status.

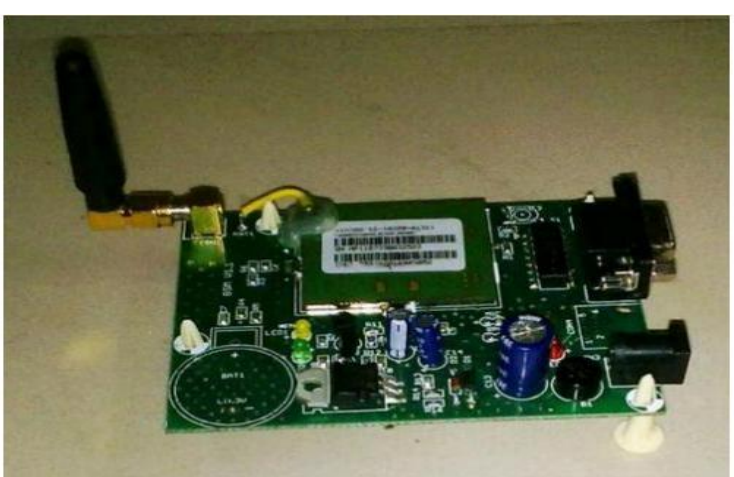

Figure 2: GSM Modem

1) Whenever GSM module receives any call, system confirms the user with password verification and allows only the legitimated user.

2) Then suitable voice message is transmitted from voice module to the user via GSM for conciliatory the numerous control actions.

3) Subsequently the authenticated user commands are transmitted to the DTMF decoder.

4) According to the command number and present status of the unit, a control action is executed.

5) If the alteration in the parameter viz. Appliance On/Off occurs the MCU gets interrupted which in turn wheels the GSM to give the status update SMS to the programmed cell phone number.

6) If GSM receives any command via call it provides this data stream to MCU via RS 232 interface. As GSM module does not store any commands, MCU treats each command as a suspended action and responds instantaneously.

7) The GSM module acknowledges only the cell phone number which is programmed on ACU primarily.

8) This eases it to send updates to only the programmed number from next communication ahead.

B. Transceiver modules: Transceiver modules can be assimilated in this system in order to share the current status of the parameters in between the two or more ACU units in the course of the continuing exchange of messages which aids MCU in execution as well as intermination of the user process.

\section{Frequency Decoder}

DTMF (Dual Tone Multiple Frequency) is a generic communication term used for the tones which are generated after pressing any keypad digit. It uses a dialing keypad of $12 / 16$ buttons producing unique tones for each button. It is termed as DTMF as two tones (dual) of dissimilar frequencies; higher $(1209 \mathrm{~Hz}$ to $1633 \mathrm{~Hz}$ ) and lower $(697 \mathrm{~Hz}$ to $941 \mathrm{~Hz}$ )nine are generated when a key is pressed.

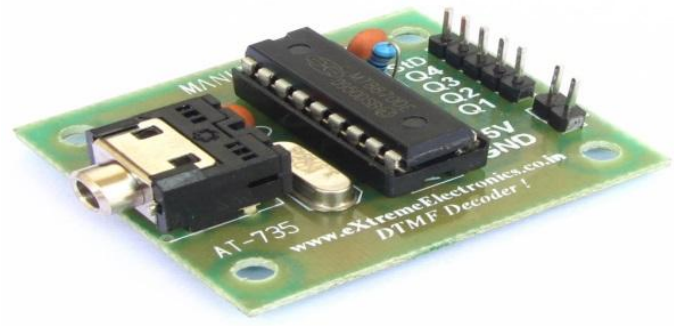

Figure 3: DTMF module 


\section{International Journal of Science and Research (IJSR)}

ISSN (Online): 2319-7064

Index Copernicus Value (2013): 6.14 | Impact Factor (2014): 5.611

\begin{tabular}{|l|c|c|c|c|}
\hline & $1209 \mathrm{~Hz}$ & $1336 \mathrm{~Hz}$ & $1477 \mathbf{~ H z}$ & $1633 \mathrm{~Hz}$ \\
\hline $697 \mathbf{~ H z}$ & 1 & 2 & 3 & $A$ \\
\hline $770 \mathrm{~Hz}$ & 4 & 5 & 6 & B \\
\hline $852 \mathrm{~Hz}$ & 7 & 8 & 9 & $C$ \\
\hline $941 \mathrm{~Hz}$ & $*$ & 0 & $\#$ & $D$ \\
\hline
\end{tabular}

\section{Voice module}

Voice module is an essential part of the system to inaugurate IVRS (Interactive Voice Response System) which affords real time communication. Rendering to the user command a suitable response which is a prerecorded voice message is produced and transmitted to user via GSM network. The voice module APR9600 used is a true single-chip voice recording IC with playback capability of 40 to 60 seconds [6].

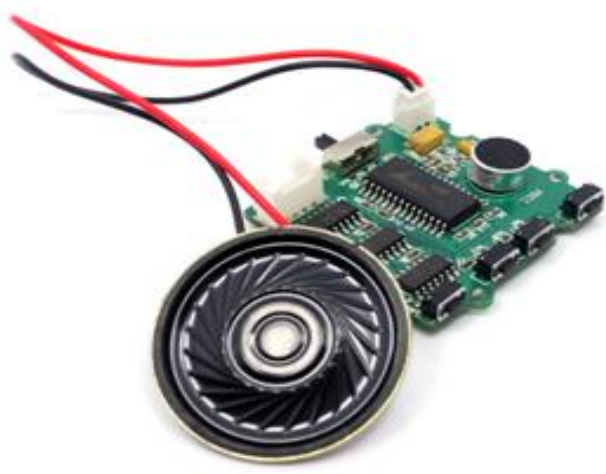

Figure 4: APR9600 module

\section{E. Control Circuit}

The control unit primarily entails of relays and its equivalent driver circuitry. The driver circuitry is inserted with transistors which are used as a switch in order to drive the relays. It is the job of the MCU to deliver the command to relay driving circuitry. Henceforth it controls the switching operation of the load by controlling the relays.

\section{Implementation of IVRS System}

Implementation of IVRS section allows the user to take suitable control action according to the present status of parameters such as fan ON/OFF, Water Tap status, AC coolness etc. Block diagram of IVRS system is as shown in Fig. 2. It also allows the user to interact with the system, so that ambiguity in performing selected tasks is avoided and the process is synchronized.

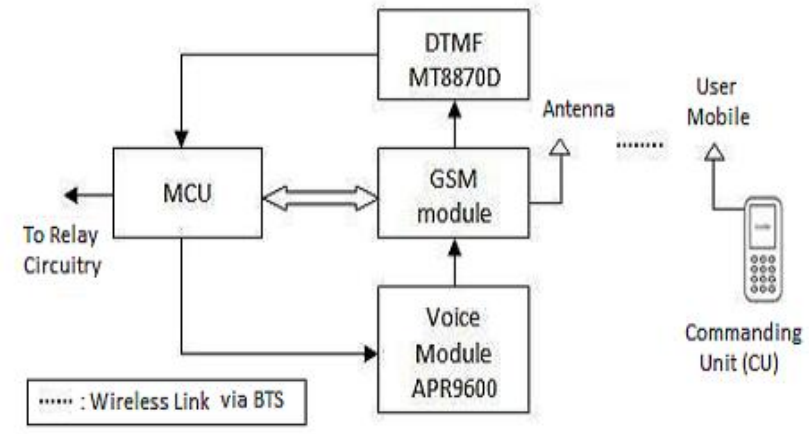

On every occasion the legitimated user calls on the SIM card no. which is inserted in GSM, the prerecorded messages are transmitted to user via GSM network radio link. In our scheme MOD8 [11] is selected which provides capacity to record 8 messages which are as follows:

- Immediately after call: Enter your password

- If password is wrong: Password wrong, try again.

- For unsuitable user input: Command error, try again.

- If password is right: Select the room to control its appliance.

- After selection of the room select the which appliance to control.

- If device is OFF: Press 1 to switch ON the device.

- If device is ON: Press 2 to switch OFF the device.

Hardware implementation of the system

The hardware execution of Appliance Control Unit which is to be implemented at the home is as shown in Fig.1. All the subcategories such as transceiver unit, Voice module, Relay Circuitry, Frequency decoder are explained in earlier sections.

\section{Implementation and Result}

The system was simulated according to the block diagram given in Fig.1. For the sake of easiness and flexibility, as an alternative of sending full commands like "Turn ON LOAD 1 ", the user can send a single number to command. A simple formula was developed that can be used to know which number to send to turn ON/OFF any load. The formula is as follows:

Load Number X 2= To turn OFF a LOAD

(Load Number X 2)-1= To Turn ON a LOAD

Load numbers can be set by the user. For instance, to turn ON load number 3, the user can send an SMS message with only the number "5" to the system, since $((3 \times 2)-1=5)$. Similarly, the user can send the number " 6 " to turn load number 3 OFF. A single controller can switch up to 8 loads. The system was simulated using Proteus v7.7 and the outcomes create to be in the estimated outlines. The $\mathrm{C}$ program used in the system was inscribed using Keil compiler and was supplementary to the simulated module.

\section{Conclusion}

This paper presents an inexpensive GSM-based interactive control system. A number of literatures related to the topic of control systems and automation were reviewed and analyzed. The system elaborately described in the above sections makes efficient use of the latest technology to aid users in order to control the operation of the desired appliance or load in real time from remote location. It also elaborates the Interactive Voice Response System (IVRS) designing integrated with Voice IC circuitry which is the unique feature of the implemented system.

Figure 2: IVRS system 


\section{References}

[1] A. Delgado, R. Picking and V. Grout, "RemoteControlled Home Automation Systems with Different", Centre for Applied Internet Research (CAIR), 2006.

[2] A. Alkar and U. Buhur, "An Internet Based Wireless Home Automation System for Multifuntional Devices", 2005.

[3] N. Sriskanthan, F. Tan and A. Karande, "Bluetooth based home automation system" ELSEVIER - Microprocessors and Microsystems, Vol. 26, pp. 281-289, 2002.

[4] R. Piyare and M. Tazil, "BLUETOOTH BASED HOME AUTOMATION SYSTEM USING CELL PHONE", IEEE International Symposium on Consumer Electronics, Vol. 15, 2011.

[5] I. Petrov, S. Seru, and S. Petrov, "HOME AUTOMATION SYSTEM", School of Engineering Science, 2011.

[6] Voice IC 9600: Datasheet for voice module: Available at: http://www.aplusinc.com.tw.

\section{Author Profile}

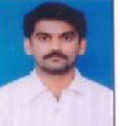

D. Prasad completed his B.E in Electronics \& Communication Engineering and M.E in the stream of Electronic Instrumentatic in the year of 2001 \&2005 from Madras University \& Andhra University respectively. He is having teaching experience of more than 13 years in various Under Graduate and Post Graduate courses. He has guided lots of students in various Under Graduate and Post Graduate Research Projects. At Present, he is working as Professor \& Vice-Principal in Ramanandatirtha Engineering College, Nalgonda, India. 